\title{
Recent work on sprite spectrum in Taiwan
}

\author{
Min-Ruey Lu ${ }^{1}$, Pei-Yu Chen ${ }^{1}$, Cheng-Ling Kuo ${ }^{1, *}$, Chien-Chung Chou ${ }^{1}$, Bin-Xing Wu ${ }^{2}$, Shinsuke Abe ${ }^{3}$, \\ Han-Tzong $\mathrm{Su}^{4}$, Rue-Ron Hsu ${ }^{4}$, Sheng-Hsiang Wang ${ }^{5}$, Neng-Huei Lin ${ }^{5}$, and Lou-Chuang Lee ${ }^{6}$ \\ ${ }^{I}$ Institute of Space Science, National Central University, Taoyuan City, Taiwan \\ ${ }^{2}$ Graduate Institute of Astronomy, National Central University, Taoyuan City, Taiwan \\ ${ }^{3}$ Department of Aerospace Engineering, Nihon University, Funabashi City, Japan \\ ${ }^{4}$ Department of Physics, National Cheng Kung University, Tainan City, Taiwan \\ ${ }^{5}$ Department of Atmospheric Sciences, National Central University, Taoyuan City, Taiwan \\ ${ }^{6}$ Institute of Earth Sciences, Academia Sinica, Taipei City, Taiwan
}

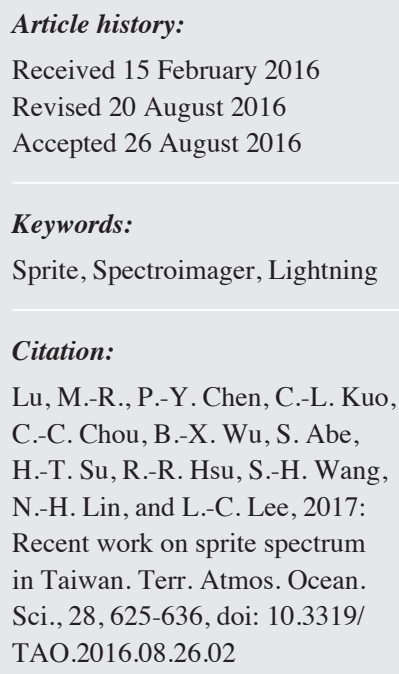

\begin{abstract}
This paper reports on the recent developments in spectroimagers for sprite campaigns in Taiwan. We first introduce two types of spectroimagers, the slit and slitless types, and discuss their advantages and shortcomings. Next we explore the instrument development and procedures undertaken for this study. In 2006, a slit spectroimager was installed for a sprite campaign and on 15 August of that year, two sprite spectra were recorded using the slit spectroimager along with seven sprites, one halo, one ELVES emission and two jets. By the end of 2015, a slitless spectroimager had been successfully constructed and was ready to conduct additional investigations. On 7 May 2016, a sprite spectrum was recorded using the slitless spectroimager. Following an examination of the calibrations (comprising detection region field of view, wavelength calibration, and response curve), data analysis, and additional calibrations (comprising elevation and azimuthal angles, atmospheric transmittance, and theoretical wavelength calculations) performed in this study, we present the results from our observed sprite spectra using the slit and slitless spectroimagers.
\end{abstract}

\section{INTRODUCTION}

A research group from the University of Minnesota was the first to demonstrate the existence of upward electrical discharges, called "sprites," on the night of 22 September 1989 (Franz et al. 1990). Later, during the sprite 1994 aircraft campaign, the first color image of the phenomena was captured, elucidating the luminosity and transient nature of its structure $(<16 \mathrm{~ms})$. Sprites typically have a red body with faint bluish tendrils extending downward, occasionally reaching the cloud top. They usually appear at altitudes of 50 - $90 \mathrm{~km}$ above the Earth's surface. The high-speed imaging of sprites with a time resolution of $0.3-1.0 \mathrm{~ms}$, halos, and ELVES emissions has been discussed by Stanley et al. (1999), Barrington-Leigh et al. (2001), and Moudry et al. (2002, 2003). Stanley et al. (1999) determined that sprites usually begin at an altitude of approximately $75 \mathrm{~km}$ and

\footnotetext{
* Corresponding author

E-mail: clkuo@jupiter.ss.ncu.edu.tw
}

simultaneously expand upward and downward from their point of origin. McHarg et al. (2007) analyzed sub millisecond images of sprites (5000 and 7200 frames s ${ }^{-1}$ ) and compiled statistics on the velocities of streamer heads, which varied between $10^{6}$ and $10^{7} \mathrm{~m} \mathrm{~s}^{-1}$. Additionally, Cummer et al. (2006) demonstrated that the long-persisting sprite beads that form at the tips of downward moving sprite streamers are attracted to and collide with other streamer channels. Higher-speed dynamic evolutions of the fine structured streamers in sprites have also been predicted by theoretical streamer models (Pasko et al. 1998; Liu and Pasko 2004, 2005; Liu et al. 2006, 2009), which are consistent with our sprite observations.

Among the main groups of sprite emissions, the molecular nitrogen first positive band $\left(\mathrm{N}_{2} 1 \mathrm{P}\right)$ was the first to be identified using an intensifier charge-coupled device (CCD) spectrograph (Mende et al. 1995). Subsequent studies further determined the vibrational excitement states of $\mathrm{N}_{2} 1 \mathrm{P}$ 
(Green et al. 1996; Hampton et al. 1996), and obtained evidence of the existence of a $\mathrm{N}_{2}{ }^{+}$Meinel band emission (Bucsela et al. 2003). More recently, 1-ms time resolution spectrograph observations were achieved (Stenbaek-Nielse and McHarg 2004). The altitude-resolved spectrum (with a 3-ms temporal resolution and approximately $3-\mathrm{nm}$ spectral resolution, from $640-820 \mathrm{~nm}$ ) revealed that the upper vibrational state population of the $\mathrm{N}_{2} 1 \mathrm{P}$ bands, $B^{3} \Pi_{g}$, varies with the altitude, which is similar to the laboratory afterglow at low pressure (Kanmae et al. 2007, 2010; Parra-Rojas et al. 2013; Stenbaek-Nielsen et al. 2013).

We discuss the recent development in spectroimagers for sprite campaigns in Taiwan. In section 2, we introduce two types of spectroimagers (slit and slitless designed), and discuss the advantages and shortcomings of each. In section 3, we list the sprite spectroimager campaign results, conducted from 2006 to the present. In section 4, we present the spectroimager calibrations (comprising detection region field of view, wavelength calibration, and response curve), data analysis, and additional calibration (comprising elevation and azimuthal angles, atmospheric transmittance, and theoretical wavelength calculations) performed in this study. Section 5 explores the observed sprite and lightning spectra results.

\section{INSTRUMENT}

In contrast to progressive scanning spectroscopy laboratory experiment applications for obtaining high-resolution wavelength, it was necessary to capture the entire low-intensity spectrum within a condensed time in this study because sprite occurrence is rare and unpredictable. The criteria required for the spectroimager sprite observations were (1) a wide field, (2) low light level, (3) short exposure time, and (4) full wavelength band scanning (visual and infrared). The slit spectrometer was originally designed to avoid the overlap of various spectral regions diffracted from diverse light sources. Conversely, the slitless spectroimager was developed to increase the likelihood of capturing a sprite in the spectroimager's field of view, and has often been used in meteor spectrum observations (Abe et al. 2004a, b; Fujiwara et al. 2004; Passas et al. 2016) because a high-resolution $(0.45 \mathrm{~nm})$ spectroimager is required to investigate the spectroscopic features of meteors (Passas et al. 2016).

Slitless spectroimagers have also been frequently used in astronomy and remote sensing (Abe et al. 2004a, b; Fujiwara et al. 2004). They are comprised of a diffracting component (transmittance grating or reflected grating), a lightpath component (mirrors or wedges), and a low-light-level sensor. The diffracting component curves the light through the grating at different angles. For a specified angle of light, the constructive wave interference enhances the light within the specified wavelength range. The destructive wave interference cancels the light outside of the specified wavelength range. The point light source curves the light through the diffracting component to the light intensity within a specified wavelength range of interest. Slitless spectroimagers are valid only for point light sources that generate the spectrum diffracted by a light source external to or within the narrow slit region. Hence, using slitless spectroimagers increases the likelihood of capturing a light point spectrum.

Compared with reflected gratings, transmittance gratings direct the light path along the instrument optical axial direction, which is easily aligned. A wedge prism was used to refract the light along the optical axis in this study because the refracted prism angle on the light path depends on the transmittance grating diffraction angle of the wavelength range of interest. Figures 1a - c illustrate the slitless spectroimager design used in this study. Table 1 presents a summary of the device optical and electrical components. The cost of the full spectroimager suite was approximately US $\$ 1000$. A commercial global positioning system module was adopted to provide an accurate timestamp on our recording system.

The slit spectroimager design is presented in Fig. 2a, and depicted schematically in Fig. 2b. One of the advantages of a slit spectroimager is that the sensor rectangular window can be fixed for light source mapping. However, this is also a shortcoming because the slit shrinks the observation window and decreases the likelihood of recording a sprite. As indicated in Fig. 2b, the first two lens components are a collimator and a slit with an adjustable width. The second and third components are the transmission grating and the CCD light sensor, respectively.

The collimator component is composed of three identical Nikon F1.4 50-mm lenses and an adjustable slit. The first lens, portrayed on the left in Fig. $2 b$, is an object lens that projects the image onto the slit. The adjusted slit component (length, $6.3 \mathrm{~mm}$ ) is opened from the center. Its size can be expanded from $0 \mathrm{~mm}$ (closed) to $6.0 \mathrm{~mm}$ (maximum clear opening). The slit width was set at $0.2 \mathrm{~mm}$ for this research. The spectrograph had a spectral resolution of approximately $6 \mathrm{~nm}$. The light from the slit, collimated by the second lens, was diffracted by the prism (one prism plus the transmission grating with 300 lines $\mathrm{mm}^{-1}$ ). The diffracted light was then focused by the third lens and finally recorded by the light sensor.

As illustrated by the dashed line in Fig. 3, the responses of the slit and slitless spectrograph systems covered the sprite infrared emissions (i.e., $\mathrm{N}_{2} 1 \mathrm{P}$ spectra). An Andor electron multiplying CCD (EMCCD) light sensor was used for the slit spectrograph. The EMCCD is a quantitative digital camera that can detect single photon events while maintaining high quantum efficiency. This is achieved through a unique electron-multiplying structure built into the sensor (see http://www.emccd.com/what_is_emccd/ for more information) and is specifically designed for time-integrated image intensified spectroscopy. 


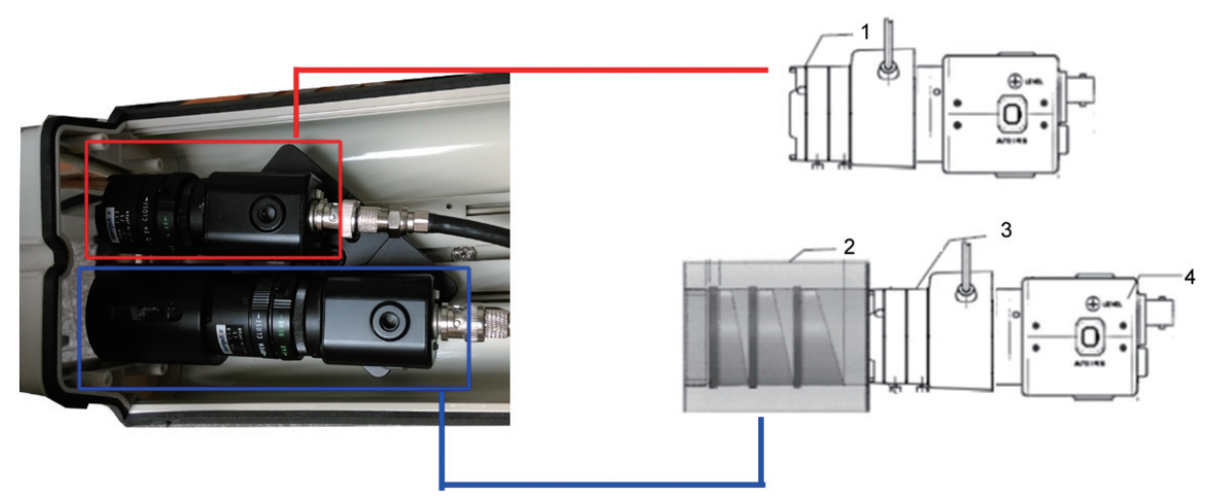

Fig. 1. Slitless spectroimager components. (Color online only)

Table 1. Slitless spectroimager optical components.

\begin{tabular}{c|cc}
\hline Item & Description & Manufacture \\
\hline 1 & Optical lens $(12 \mathrm{~mm})$ & Computar \\
2 & Diffracting component & Unice E-O \\
3 & Optical lens $(8 \mathrm{~mm})$ & Computar \\
4 & Monochrome CCD & Watec \\
\hline
\end{tabular}

(a)

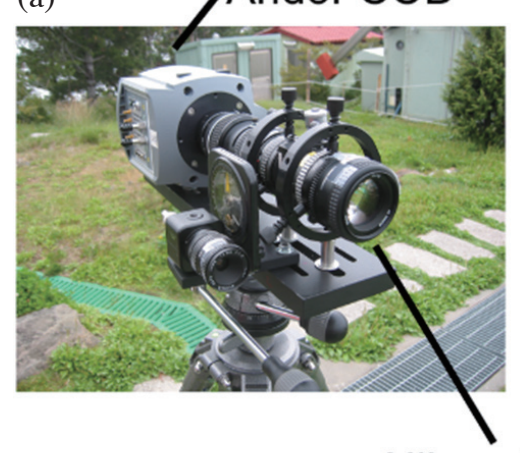

Andor CCD

Nikon Camera (b)

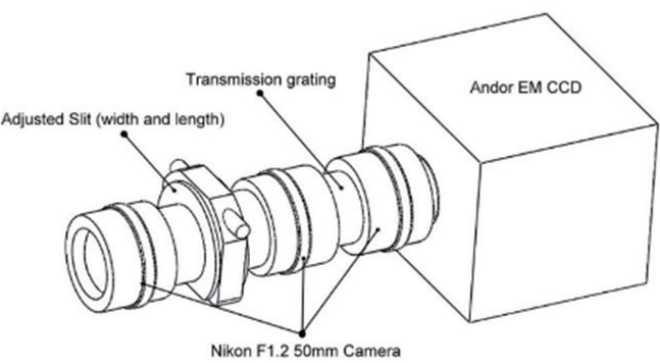

Nikon F1.2 $50 \mathrm{~mm}$ Camera

Fig. 2. (a) Photograph of the slit spectroimager, and (b) optical layouts of the slit spectroimager. (Color online only)

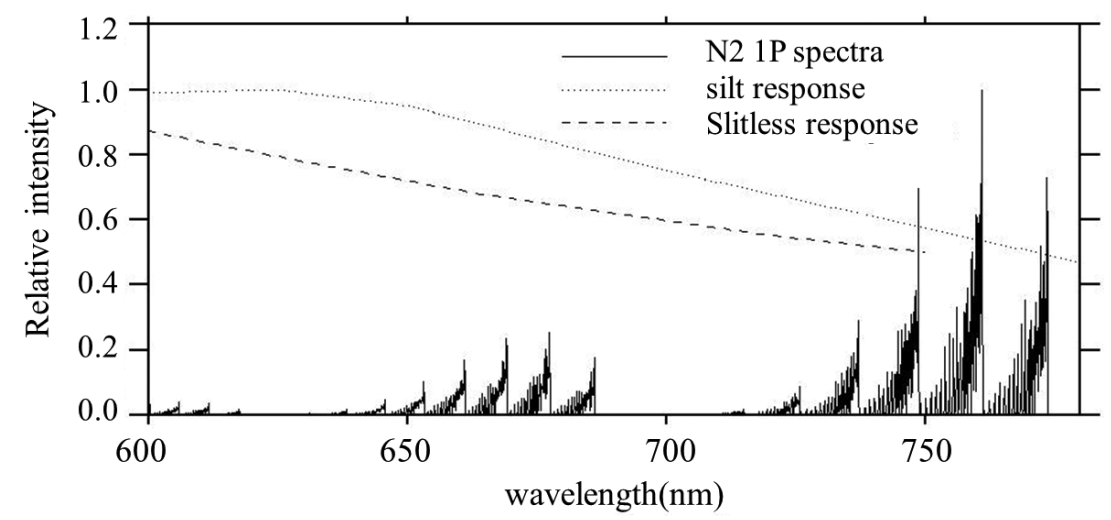

Fig. 3. Simulated spectra for $\mathrm{N}_{2} 1 \mathrm{P}$ band emissions (solid lines), and relative responses of the slit spectroimager (dotted line) and slitless spectroimager (dash line). 


\section{OBSERVATIONS}

We conducted a sprite campaign at the Lulin Observatory in Taiwan during the summer of 2006. On 15 August 2006, a total of seven sprites, one halo, one ELVES emission, and two jets were recorded using the low-light-level CCD system. Of these events, two sprites and one lightning spectrum were recorded in a slit spectroimager, which has a narrower field of view than do low-light-level CCD systems.

From 2010 - 2012, the ground campaign from Lulin Observatory and the National Central University (NCU) campaign were established and recorded by Shinsuke Abe and BinXing Wu. A total of 158 events were analyzed, with 140 sprites, 6 ELVES emissions, 5 gigantic jets, and 2 blue jets recorded (Table 2); the events per month between 2010 and 2012 are summarized in Table 3. The recording of transient luminous events (TLEs) began in May (Table 3), coinciding with Taiwan's monsoon season, which starts in early May and ends in late September [Central Weather Bureau (CWB)]. The monsoon season is accompanied with typhoons, especially from June to September, that cause a seasonal wind reversal. Hence, the climate during the monsoon season tends to produce thunderstorms with sprites. According to the CWB, typhoons are most common in August; correspondingly, the number of TLEs recorded in August and September was higher than that in other months. In contrast to our expectations, the number of events in November 2011 was also very high. The projected location of detected TLEs near Taiwan and the spatial distribution of recorded events from 2010 - 2012 at the Lulin Observatory are presented in Fig. 4.

Between 2012 and 2014, the sprite ground observatory was tested from three stations: the NCU station, the NCU Taiwan Coastal Observation and Assessment Station

Table 2. Results of the 2010 - 2012 Lulin Observatory ground campaign.

\begin{tabular}{|c|c|c|c|c|c|c|c|c|}
\hline Type & May & June & July & August & September & October & November & Sum \\
\hline Blue jets & & & & 1 & & & 1 & 2 \\
\hline Elves & & & & 4 & 1 & 1 & & 6 \\
\hline Gigantic jet & & & & 1 & 4 & & & 5 \\
\hline Halos & & & & 5 & 1 & & & 6 \\
\hline Sprites & 38 & 1 & 4 & 24 & 29 & 7 & 30 & 133 \\
\hline Sprite + elve & & & & & 1 & & & 1 \\
\hline Sprite + halo & & & & 2 & 3 & & & 5 \\
\hline Total & 38 & 1 & 4 & 37 & 39 & 8 & 31 & 158 \\
\hline
\end{tabular}

Table 3. Number of events detected from the Lulin Observatory between 2010 and 2012 .

\begin{tabular}{|c|c|c|c|c|}
\hline Month Year & 2010 & 2011 & 2012 & Month total \\
\hline 05 & & 17 & 21 & 38 \\
\hline 06 & & 1 & & 1 \\
\hline 07 & & 4 & & 4 \\
\hline 08 & 32 & 5 & & 37 \\
\hline 09 & 34 & 5 & & 39 \\
\hline 10 & 2 & 6 & & 8 \\
\hline 11 & & 31 & & 37 \\
\hline Year total & 68 & 69 & 21 & 158 \\
\hline
\end{tabular}

\section{Event Distribution}

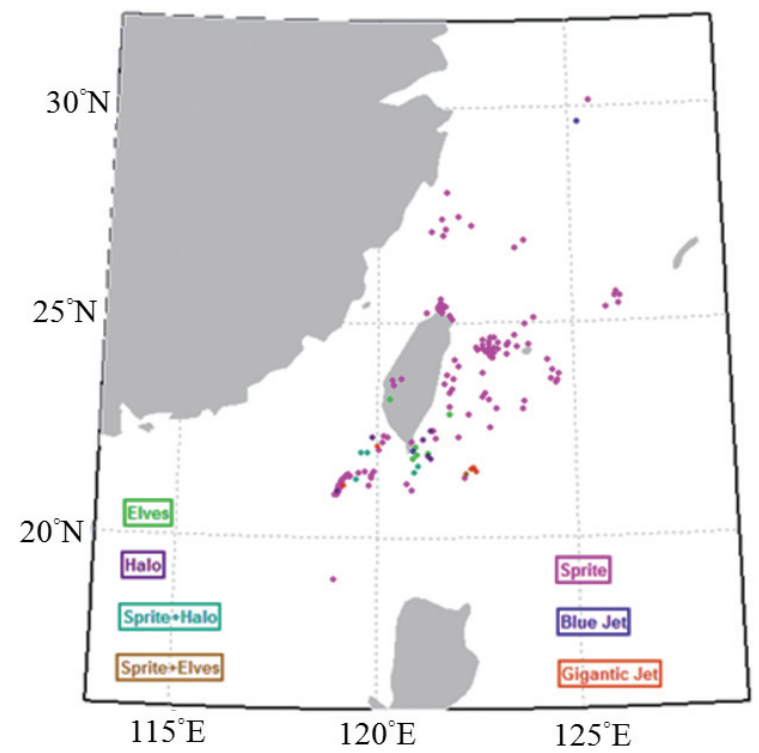

Fig. 4. Spatial distribution of events recorded at the Lulin Observatory from 2010 - 2012. (Color online only) 
(TaiCOAST), and the Lulin Observatory. In 2015, the Lulin Observatory was selected for a spectroimager campaign, and a slitless spectroimager with a Watec-910HX detector was installed in April 2015. However, during the first test run, the auto recording system had difficulty recording both a sprite and its spectrum simultaneously; thus, in October 2015; the composite image (picture-in-picture) method using video synthesis was adopted. The images acquired from the slitless spectroimager were displayed on a full screen, and the associated sprite image was displayed in a small window at the lower right-hand corner; this technique successfully enabled synchronous video recording of the sprite and associated spectral images.

\section{INSTRUMENT CALIBRATION}

\subsection{Optical Laboratory for Calibration}

Figure 5 illustrates the optical layout for the spectroimager calibration experiment. Table 4 presents the optical components. Halogen lamps were used as the calibrated visual light source, although Hamamatsu Xenon lamps are recommended for future studies. The wideband light was first diffracted into narrowband light using a monochromator. The point light source was next placed on the focal point of the parabolic mirror where the optical flat mirror folds the light path into a shorter length on a small optical board. The parabolic and optical flat mirrors were then used to project the point source from the iris diaphragm to the light source at an infinite distance. subsequently, the source light alignment was tested using the collimator, and the detection region field of view, wavelength, and response curve of the spectroimager were calibrated in the optical laboratory, as described by Harris $(2000,2003)$.

\subsection{Elevation and Azimuthal Angle}

The azimuthal and elevation angles of the recorded stars were calibrated in the CCD spectrograph. The uncovered star fields were compared with the star field in the astronomy software Stellarium. Figure 6a depicts the stars that were selected in this study for calibrating the azimuthal and elevation angles. The azimuthal and elevation star angles from Stellarium were subsequently fitted with a linear function to determine the angles of each recorded image frame pixel (Figs. 6b and c).

\subsection{Wavelength Calibration}

Several methods can be employed to calibrate a spectroimager wavelength. For slitless spectroimagers, the simplest approach to determine the wavelength position on an image frame is to identify the characteristic wavelength of the recorded spectrum, because the expanded spectrum de- pends on the incident angle of the point light source. The position of the wavelength subsequently changes as a function of this incident angle. For slit spectroimagers, this angle can be limited by the width of the slit. Therefore, wavelength calibrations in a laboratory experiment are consistent with the observed spectrum. For laboratory experiments, a standard lamp (e.g., Newport 6035) is sufficient for calibrating the wavelength of recorded spectra before observation.

\subsection{Atmospheric Transmittance}

Atmospheric transmittance along the sight line affects the percentage of band emissions $\left(B_{k}\right)$, and is calculated using Lambert's law:

$T(\lambda, h, L)=\exp \left[-\sum_{x} \sigma_{x}(\lambda) \mathrm{D}_{x}(h, L)\right]$

where $\sigma_{x}(\lambda)$ is the absorption cross-section for the major atmospheric species, namely $\mathrm{O}_{2}$ absorption, $\mathrm{O}_{3}$ absorption, and molecular Rayleigh scattering $\left(\mathrm{N}_{2}, \mathrm{O}_{2}\right)$. The data for $\mathrm{O}_{2}$, $\mathrm{O}_{3}$, and molecular Rayleigh scattering were retrieved from Jursa (1985) and Minschwaner et al. (1992). In this study, the absorption cross-section of $\mathrm{N}_{2}$ was minor (185 - $\left.800 \mathrm{~nm}\right)$, and its effect on transmittance was negligible.

Column density $\left[\left(\mathrm{D}_{x}(h, L)\right]\right.$ was calculated by integrating the densities of $\mathrm{N}_{2}, \mathrm{O}_{2}$, and $\mathrm{O}_{3}$ from the observation site to the assumed altitude $(h)$ of a TLE:

$\mathrm{D}_{x}\left(h_{T L E}, L\right)=\int_{F S-2}^{h_{T L E}} n_{x}(s) d s$

where the total length of the path between the observation site and the TLE is $L$. The $\mathrm{N}_{2}$ and $\mathrm{O}_{2}$ densities were computed using the MSIS model (Hedin 1991), whereas the overnight $\mathrm{O}_{3}$ density profile at an altitude between 18 and $100 \mathrm{~km}$ was measured using the Michelson interferometer for passive atmospheric sounding (Verronen et al. 2005). The $\mathrm{O}_{3}$ region below $100 \mathrm{~km}$ accounted for most of the $\mathrm{O}_{3}$ absorption in the calculated atmospheric extinction. Figure 7 illustrates the distance transmittance $(200,300,400$, and $500 \mathrm{~km})$ between the observation site (altitude approximately $2862 \mathrm{~m}$ ) and the sprite.

\subsection{Theoretical Wavelength Calculation}

Atmospheric gas heating produces two problems: cold plasma in the middle atmosphere (in the form of TLEs) and hot plasma (in the form of lightning). Thus, the plasma chemistry model for cold plasma (Sentman et al. 2008; Kuo et al. 2011) was applied to study the associated radiative emissions that accompany atmospheric discharge $\left(\mathrm{N}_{2}\right.$ 1P/2P/LBH, $\mathrm{N}_{2}{ }^{+}$Meinel/1N, atmospheric $\mathrm{O}_{2}$, and $\mathrm{O}_{2}{ }^{+} 1 \mathrm{~N}$ bands) (Gordillo-Vázquez et al. 2011, 2012). Notably, the 


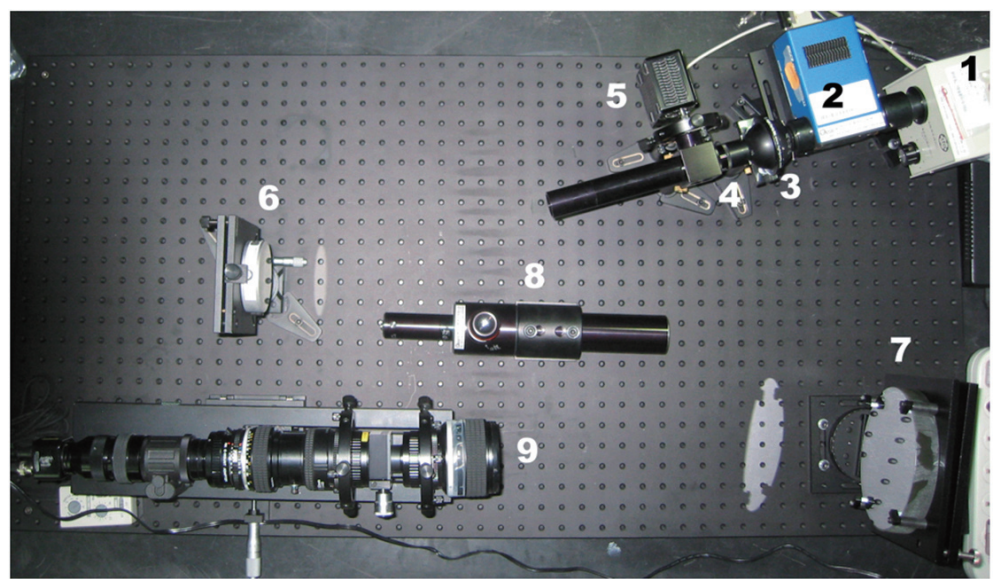

Fig. 5. Optical table to calibrate the field of view, wavelength, and optical response of a spectroimager. The numbers correspond with the optical components listed in Table 4. (Color online only)

Table 4. Optical components used in the optical laboratory for calibration. The number for each component appears in Fig. 5.

\begin{tabular}{c|cc}
\hline Item & Description & Manufacturer \\
\hline 1 & Light source & TAIWAN FIBEROPTICS \\
2 & Monochromator & CVI \\
3 & Integrating Sphere & Light Ports \\
4 & Iris Diaphragm & Edmund \\
5 & Calibrated microspectrometer $(250-950 \mathrm{~nm})$ & BWTEK \\
6 & Optical Flat Mirrors - 3.0”D Dia: $76.2 \mathrm{~mm}$ Thickness: $19.1 \mathrm{~mm}$ & Edmund \\
7 & Parabolic Mirrors EFL $=1219.2 \mathrm{~mm}$ Dia $152.4 \mathrm{~mm}$ & Edmund \\
8 & Auto Collimator \pm 55 arc min, per 5 arc minute gradations & Edmund \\
9 & Spectroimager & - \\
\hline
\end{tabular}

(a)

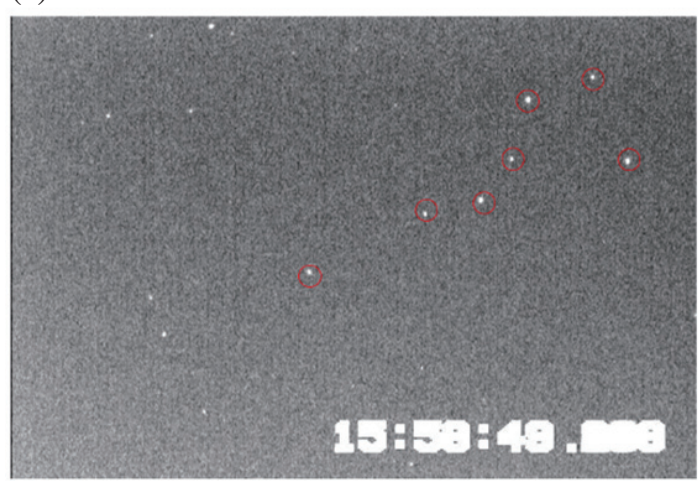

(b)
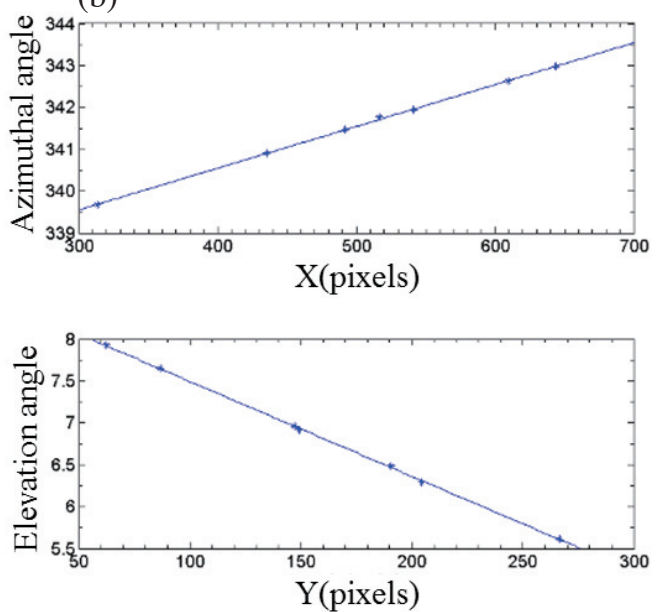

Fig. 6. Calibration of (a) azimuthal and (b) elevation angles using stars selected from Stellarium. (Color online only) 

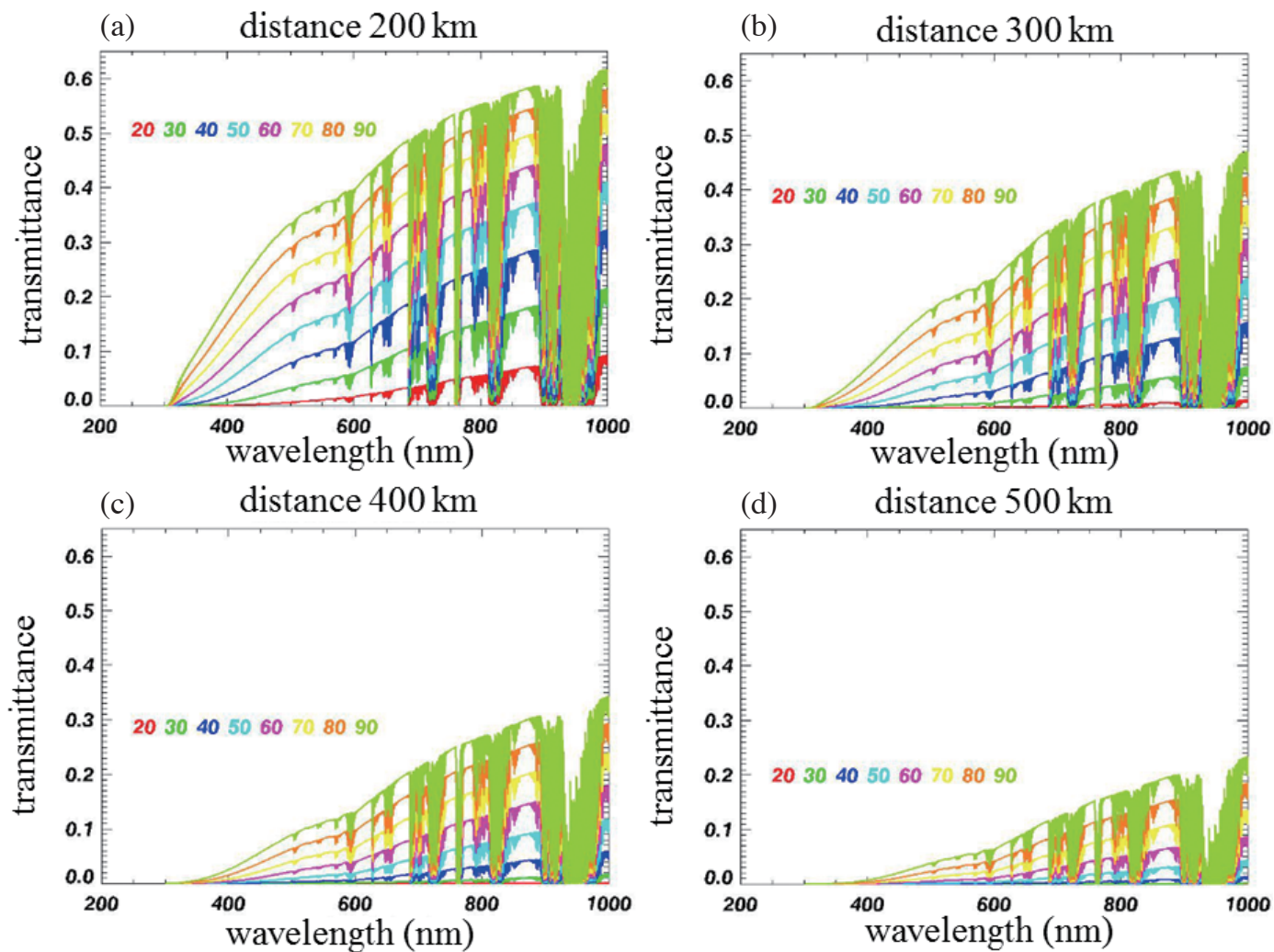

(d) distance $500 \mathrm{~km}$

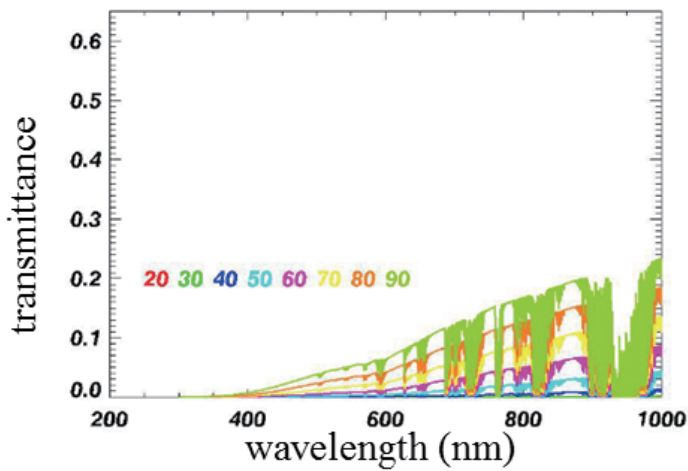

Fig. 7. Atmospheric transmittance at (a) $200 \mathrm{~km}$, (b) $300 \mathrm{~km}$, (c) $400 \mathrm{~km}$, and (d) $500 \mathrm{~km}$ between the observation site and the assumed sprite altitude (20 - $90 \mathrm{~km}$, with increments of $10 \mathrm{~km}$ ), which are labeled with different colors. (Color online only)

specified energy level $\left(E_{e, v, J}\right)$ of the upper or lower state of a given emission band in $\mathrm{N}_{2}$ can be expressed as the sum of the electron term energy $\left(T_{e}\right)$, the vibrational term energy $[G(v)]$, and the rotational term energy $\left[F_{v}(J)\right]$, as follows:

$$
\begin{aligned}
G(v) \approx & \omega_{e}\left(v+\frac{1}{2}\right)-\omega_{e} \chi_{e}\left(v+\frac{1}{2}\right)^{2}+ \\
& \omega_{e} y_{e}\left(v+\frac{1}{2}\right)^{3}+\omega_{e} z_{e}\left(v+\frac{1}{2}\right)^{4} \\
F_{v}(J) \approx & {\left[B_{e}-\alpha_{e}\left(v+\frac{1}{2}\right)\right] J(J+1)-} \\
& {\left[D_{e}+\beta_{e}\left(v+\frac{1}{2}\right)\right] J^{2}(J+1)^{2} }
\end{aligned}
$$

where $G(v)$ and $F_{v}(J)$ are fitted using Klein-Dunham polynomial expansions for the specified vibrational quantum number $v$ and the specified rotational quantum number $J$ of the energy level (Lofthus and Krupenie 1977). The formula for calculating the vibrational and rotational term energies $G(v)$ and $F_{v}(J)$ can be truncated into four and two terms, respectively, and approximating the related parameters (namely, $T_{e}, \omega_{e}, x_{e}, y_{e}, z_{e}, \alpha_{e}, \beta_{e}, B_{e}$, and $D_{e}$ ) for $1 \mathrm{PN}_{2}, 2 \mathrm{PN}_{2}, 1 \mathrm{NN}_{2}{ }^{+}$ (Herzberg 1950; Krupenie 1972; Naghizadeh-Kashani et al. 2002), and $\mathrm{N}_{2}$ LBH (NIST Chemistry WebBook).

\section{RESULTS}

\subsection{The Slit Spectroimager}

Figures $8 \mathrm{a}$ and b depict a sprite recorded at 15:58:49 on 15 October 2006, by 12- and 50-mm lenses, respectively, with an exposure time of approximately $17 \mathrm{~ms}$ for both image frames. Figure 9a illustrates the possible projected position of a sprite recorded in color and enhanced with infrared imaging using multifunctional transport satellites. The distance between the sprite and the observation site is approximately $400 \mathrm{~km}$. Figure $9 \mathrm{~b}$ displays additional sprite images recorded using the $50-\mathrm{mm}$ lens, wherein the column sprite heights were estimated to be $50-85 \mathrm{~km}$ and the spectroimager slit was estimated at a height of $55.4-61.4 \mathrm{~km}$. Figure 9c presents a wide-field sprite recording acquired using a $12-\mathrm{mm}$ lens. Figure $9 \mathrm{~d}$ shows the observed image recorded by the Andor EMCCD. The images indicate that the sprites acquired through the slit were converted into intensity profiles as a function of the wavelength. A recorded sprite spectrum, where the $\mathrm{N}_{2} 1 \mathrm{P}$ band wavelength is indicated by the difference between the vibrational number of the upper states and that of the lower states, is shown in Fig. 10. The sprite spectrum was measured between 600 and $780 \mathrm{~nm}$, and the observed spectrum included all of the major 
$\mathrm{N}_{2}$ 1P band emission characteristics.

Figure 11 shows composite images of the lightning observed at 01:52:34 on 25 October 2006, which was recorded by both the 50-mm lens (Fig. 11a) and the Andor EMCCD camera (Fig. 11b). Figure 11c indicates the possible position of the lightening over the thunderstorm and Fig. 11d depicts the recorded lightning spectrum where the $\mathrm{N}_{2} 1 \mathrm{P}$ band and the OI line (744.2 and $777.4 \mathrm{~nm}$, respectively) are located.

\subsection{The Slitless Spectroimager}

Figure 12 depicts a sprite recorded at 02:12:58 on 7 May 2016 (acquired using a 12-mm lens) and the spectra recorded using the slitless spectroimager. The relative spectrum intensity was calibrated according to the Jupiter spectrum wavelength intensity, recorded on the same night and determined by four absorption lines $\left(\mathrm{H}_{\alpha} 656.3 \mathrm{~nm}, \mathrm{H}_{\beta} 486.1 \mathrm{~nm}, \mathrm{H}_{\gamma}\right.$
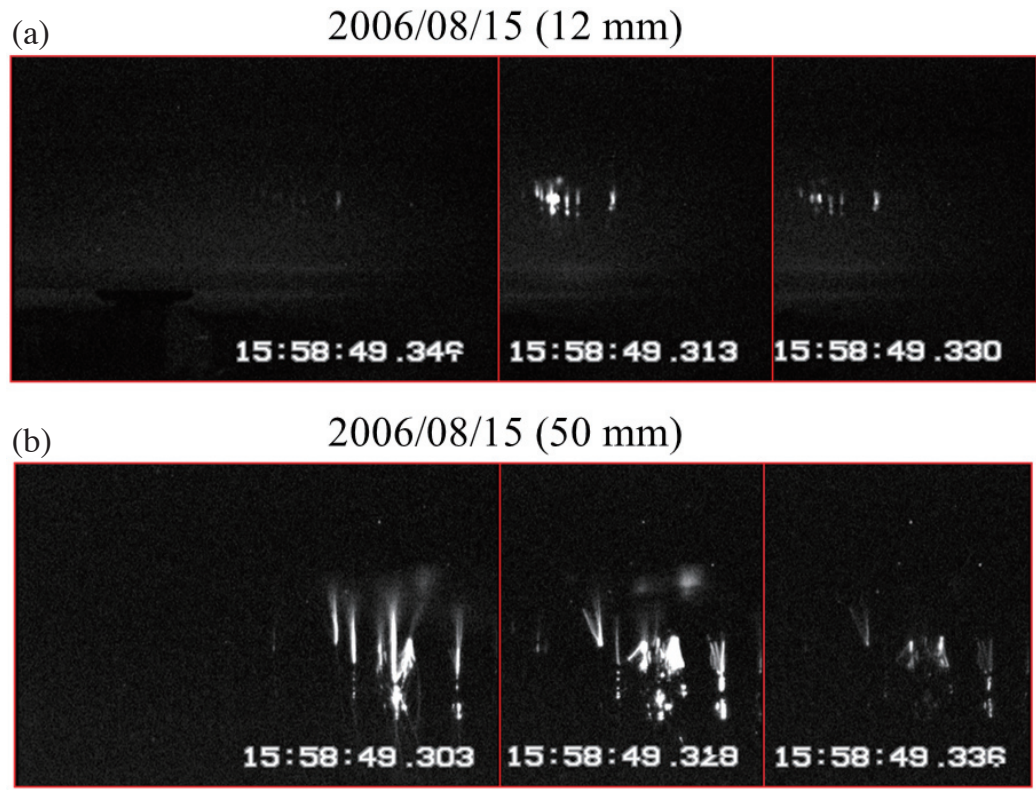

Fig. 8. Sprite recorded at 15:58:49 on 15 October 2006, captured using a (a) 12-mm lens and (b) 50-mm lens. (Color online only)

(a) MTSAT IR Image

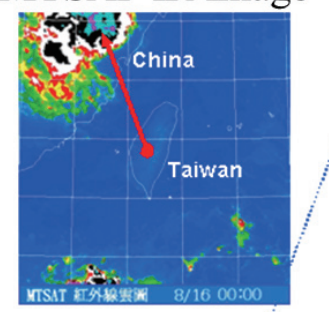

(b) Sprite 50mm camera image at 06/08 11:58:49

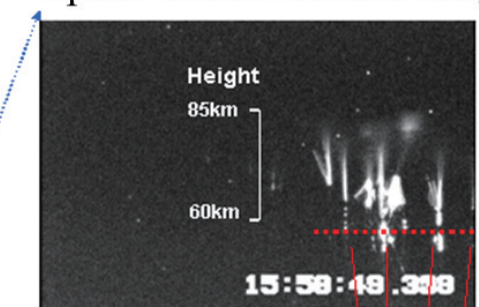

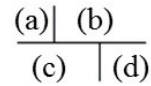

Slit width $\sim 9 \mathrm{~nm}$

Height 55.1-61.4km
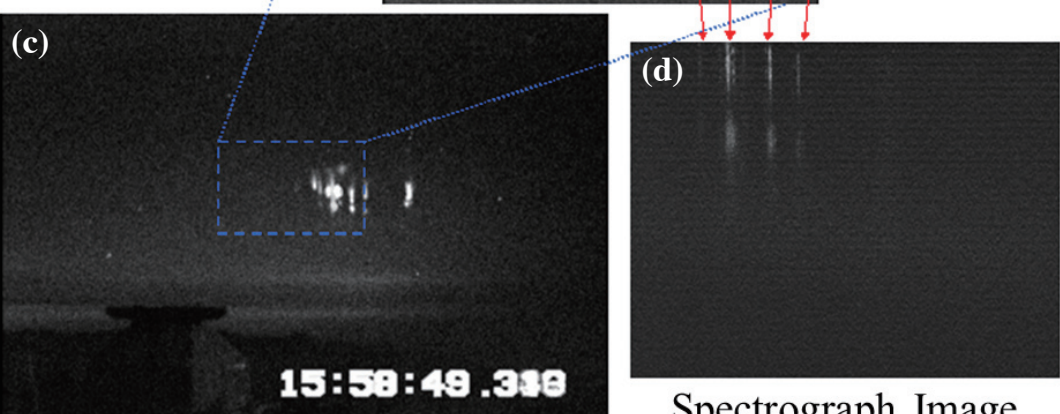

Spectrograph Image

$12 \mathrm{~mm}$ camera Image

Fig. 9. Lulin Observatory sprite campaign from the summer of 2006 in Taiwan, including (a) the weather map with the location of the Lulin Observatory (red dot), (b) the sprite recorded at 15:58:49 on 15 October 2006, acquired using a 50-mm lens, (c) the same sprite, acquired using a 12-mm lens, and (d) the spectrum recorded by the slit spectroimager. (Color online only) 


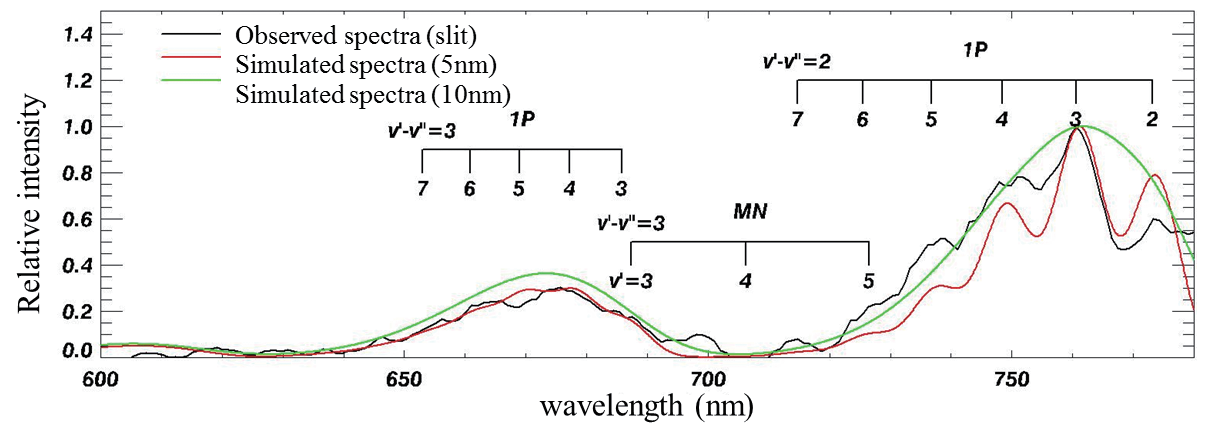

Fig. 10. Sprite spectrum (solid line) recorded by the slit spectroimager. The marks indicate that the vibrational transitions of the $\mathrm{N}_{2} 1 \mathrm{P}$ band, and the spectral resolution is approximately $6 \mathrm{~nm}$. (Color online only)
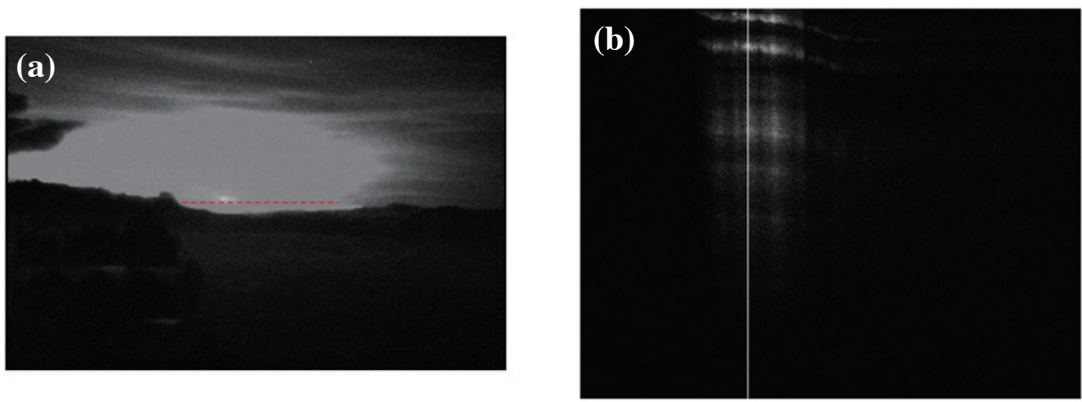

(c)

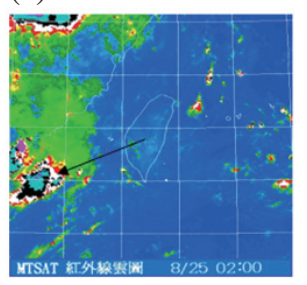

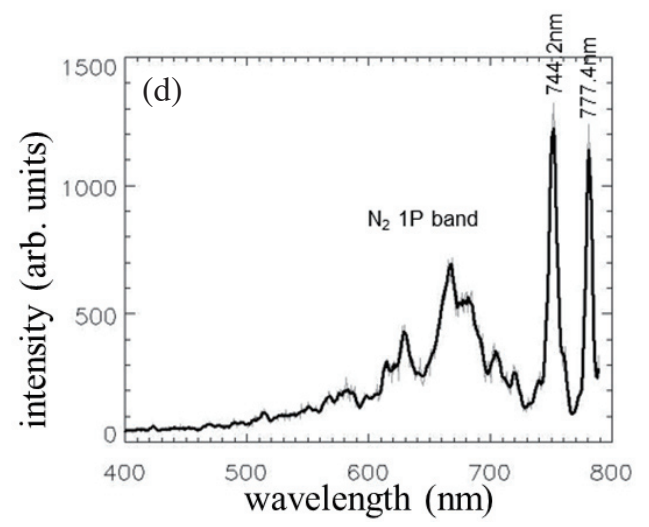

Fig. 11. Lightning recorded at 01:52:34 on 25 October 2006. (a) Lightning image, (b) the spectrum recorded by the slit spectroimager, (c) the weather map (black arrow indicates the direction from the Lulin Observatory to the lightning location), and (d) the recorded spectrum of lightning. (Color online only)
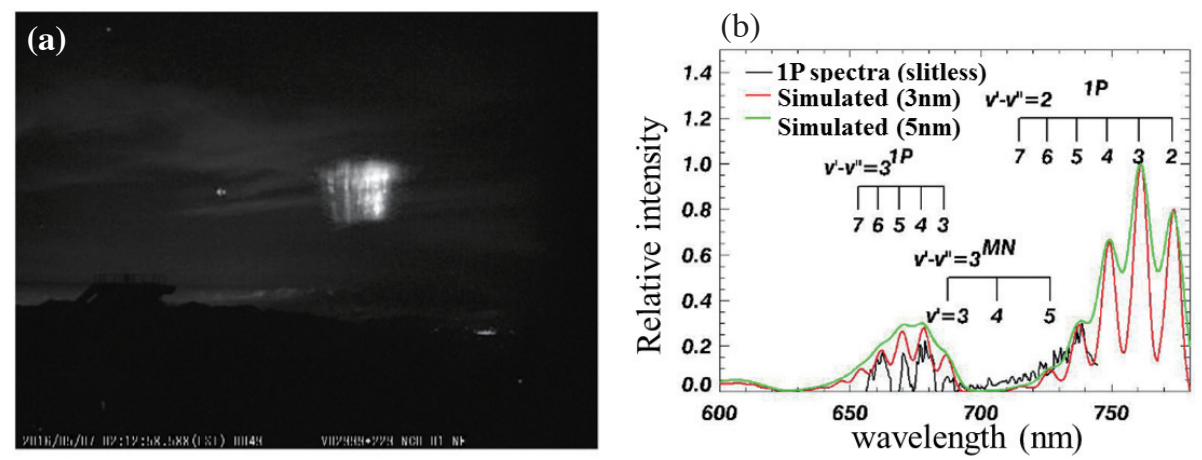

Fig. 12. (a) Sprite image taken acquired using a 12-mm lens at 02:12:58 on 7 May 2016, and (b) spectrum recorded using the slitless spectroimager. (Color online only) 
434.1, and $\left.\mathrm{H}_{\delta} 410.2 \mathrm{~nm}\right)$. As indicated by the dashed line in Fig. 3, the slitless spectroimager response can be determined through the modeling Jupiter spectra intensity and the atmospheric transmittance at a specified elevation angle. Notably, the maximum response value is $750 \mathrm{~nm}$ because that was the maximum Jupiter spectrum wavelength recorded by our slitless spectrograph. However, the wavelength limit can be improved by integrating more Jupiter spectra image frames to enhance the spectral intensity above $750 \mathrm{~nm}$.

The recorded spectra resolution acquired by the slitless spectroimager was approximately $3 \mathrm{~nm}$, compared with the simulated sprite spectra with two slit functions (widths of 3 and $5 \mathrm{~nm}$ ). As the recorded sprite spectra presented in Fig. 10 (slit spectrograph) and Fig. 12 (slitless spectrograph) demonstrate, the $\mathrm{N}_{2}$ Meinel band can be mixed with the dominant $\mathrm{N}_{2}$ 1P band emission, which peaks between 650 and $700 \mathrm{~nm}$. Our results are not comparable with the 1-nm wavelength resolution spectra observed by other researchers (Kanmae et al. 2007, 2010; Stenbaek-Nielsen et al. 2013). However, our slitless spectroimager has many advantages including its compact and lightweight size, low cost (US\$1500), and simple, easy assembly process. Additionally, the proposed slitless spectroimager is appropriate for education, particularly among college or high school students conducting sprite research.

\section{SUMMARY}

In addition to instrument development and sprite observation, the related spectroimager calibration and data analysis work, including optical experiment design, are reported in this paper. The spectroimager calibrations included the detection region field of view, wavelength calibration and spectroimager response curve. The elevation and azimuthal angles of the observation site, atmospheric transmittance and theoretical wavelength calculations for spectrum analysis were discussed.

In addition, we explored the recent development in spectroimagers in Taiwan for sprite campaigns. Notably, a slit spectroimager was developed and used for sprite campaigns in 2006. In that year, two sprite spectra were recorded using our spectroimager along with seven sprites, one halo, one ELVES emission and two jets. By the end of 2015, a slitless spectroimager was ready to conduct additional investigations, and during the subsequent 2016 sprite campaign, a sprite spectrum was successfully recorded using the slitless spectroimager. Slitless spectroimagers are substantially more cost-effective than slit spectroimagers and their assembly process is simple, which are critical advantages for young students conducting their first sprite studies.

Acknowledgements This work was supported in part by grants (MOST 103-2111-M-008-021, MOST 104-2111-M008-023, and MOST 105-2111-M-008-007) from Ministry of Science and Technology in Taiwan. We thank Ming-Han Chou, Ying-Xuan Chen, Yen-Chan Chen, and Wei-Chen Lai for their assistance in these observations.

\section{REFERENCES}

Abe, S., J. Borovicka, S. Sugimoto, and M. Yamamoto, 2004a: TV spectroscopy of persistent trains during 2001 Leonid meteor storm over Japan. In: Paillé, J. P. (Ed.), 35th COSPAR Scientific Assembly, 18 - 25 July 2004, Paris, France.

Abe, S., N. Ebizuka, H. Murayama, K. Ohtsuka, S. Sugimoto, M. Y. Yamamoto, H. Yano, J. I. Watanabe, and J. Borovička, 2004b: Video and Photographic Spectroscopy of 1998 and 2001 Leonid Persistent Trains from 300 TO 930 nm. Earth Moon Planets, 95, 265-277, doi: 10.1007/s11038-005-9031-0. [Link]

Barrington-Leigh, C. P., U. S. Inan, and M. Stanley, 2001: Identification of sprites and elves with intensified video and broadband array photometry. J. Geophys. Res., 106, 1741-1750, doi: 10.1029/2000JA000073. [Link]

Bucsela, E., J. Morrill, M. Heavner, C. Siefring, S. Berg, D. Hampton, D. Moudry, E. Wescott, and D. Sentman, 2003: $\mathrm{N}_{2}\left(B^{3} \Pi_{\mathrm{g}}\right)$ and $\mathrm{N}_{2}{ }^{+}\left(A^{2} \Pi_{\mathrm{u}}\right)$ vibrational distributions observed in sprites. J. Atmos. Sol.-Terr. Phys., 65, 583590, doi: 10.1016/S1364-6826(02)00316-4. [Link]

Cummer, S. A., N. Jaugey, J. Li, W. A. Lyons, T. E. Nelson, and E. A. Gerken, 2006: Submillisecond imaging of sprite development and structure. Geophys. Res. Lett., 33, L04104, doi: 10.1029/2005GL024969. [Link]

Franz, R. C., R. J. Nemzek, and J. R. Winckler, 1990: Television Image of a Large Upward Electrical Discharge Above a Thunderstorm System. Science, 249, 48-51, doi: 10.1126/science.249.4964.48. [Link]

Fujiwara, Y., M. Ueda, M. Sugimoto, T. Sagayama, and S. Abe, 2004: TV Observation of the Daytime Meteor Shower. The Arietids. Earth Moon Planets, 95, 595600, doi: 10.1007/s11038-005-5040-2. [Link]

Gordillo-Vázquez, F. J., A. Luque, and M. Simek, 2011: Spectrum of sprite halos. J. Geophys. Res., 116, A09319, doi: 10.1029/2011JA016652. [Link]

Gordillo-Vázquez, F. J., A. Luque, and M. Simek, 2012: Near infrared and ultraviolet spectra of TLEs. J. Geophys.Res., 117, A05329, doi: 10.1029/2012JA017516. [Link]

Green, B. D., M. E. Fraser, W. T. Rawlins, L. Jeong, W. A. M. Blumberg, S. B. Mende, G. R. Swenson, D. L. Hampton, E. M. Wescott, and D. D. Sentman, 1996: Molecular excitation in sprites. Geophys. Res. Lett., 23, 2161-2164, doi: 10.1029/96GL02071. [Link]

Hampton, D. L., M. J. Heavner, E. M. Wescott, and D. D. Sentman, 1996: Optical spectral characteristics of sprites. Geophys. Res. Lett., 23, 89-92, doi: 10.1029/95GL03587. [Link] 
Harris, S., 2000: ISUAL Imager Science Performance Test Report, Space Sciences Laboratory, UCB, Berkeley, CA, Doc. 8988-W7 rev B.

Harris, S., 2003: ISUAL Spectrophotometer Science Performance Test Report, Space Sciences Laboratory, UCB, Berkeley, CA, Doc. 8998W7 rev B.

Hedin, A. E., 1991: Extension of the MSIS Thermosphere Model into the middle and lower atmosphere. J. Geophys. Res., 96, 1159-1172, doi: 10.1029/90JA02125. [Link]

Herzberg, G., 1950: Molecular Spectra and Molecular Structure, Van Nostrand, New York.

Jursa, A. S., 1985: Handbook of Geophysics and the Space Environment, 4th Ed., Air Force Geophysics Laboratory, Hanscom AFB, MA, 25 pp.

Kanmae, T., H. C. Stenbaek-Nielsen, and M. G. McHarg, 2007: Altitude resolved sprite spectra with $3 \mathrm{~ms}$ temporal resolution. Geophys. Res. Lett., 34, L07810, doi: 10.1029/2006GL028608. [Link]

Kanmae, T., H. C. Stenbaek-Nielsen, M. G. McHarg, and R. K. Haaland, 2010: Observation of sprite streamer head's spectra at 10,000 fps. J. Geophys. Res., 115, A00E48, doi: 10.1029/2009JA014546. [Link]

Krupenie, P. H., 1972: The spectrum of molecular oxygen. J. Phys. Chem. Ref. Data, 1, 423-534, doi: 10.1063/1.3253101. [Link]

Kuo, C. L., S. C. Chang, L. J. Lee, T. Y. Huang, A. B. Chen, H. T. Su, R. R. Hsu, D. D. Sentman, H. U. Frey, S. B. Mende, Y. Takahashi, and L. C. Lee, 2011: The $762 \mathrm{~nm}$ emissions of sprites. J. Geophys. Res., 116, A01310, doi: 10.1029/2010JA015949. [Link]

Liu, N. and V. P. Pasko, 2004: Effects of photoionization on propagation and branching of positive and negative streamers in sprites. J. Geophys. Res., 109, A04301, doi: 10.1029/2003JA010064. [Link]

Liu, N. and V. P. Pasko, 2005: Molecular nitrogen LBH band system far-UV emissions of sprite streamers. Geophys. Res. Lett., 32, L05104, doi: 10.1029/2004GL022001. [Link]

Liu, N., V. P. Pasko, D. H. Burkhardt, H. U. Frey, S. B. Mende, H. T. Su, A. B. Chen, R. R. Hsu, L. C. Lee, H. Fukunishi, and Y. Takahashi, 2006: Comparison of results from sprite streamer modeling with spectrophotometric measurements by ISUAL instrument on FORMOSAT-2 satellite. Geophys. Res. Lett., 33, L01101, doi: 10.1029/2005GL024243. [Link]

Liu, N. Y., V. P. Pasko, K. Adams, H. C. Stenbaek-Nielsen, and M. G. McHarg, 2009: Comparison of acceleration, expansion, and brightness of sprite streamers obtained from modeling and high-speed video observations. J. Geophys. Res., 114, A00E03, doi: 10.1029/2008JA013720. [Link]

Lofthus, A. and P. H. Krupenie, 1977: The spectrum of molecular nitrogen. J. Phys. Chem. Ref. Data, 6, 113-307, doi: 10.1063/1.555546. [Link]

McHarg, M. G., H. C. Stenbaek-Nielsen, and T. Kammae, 2007: Observations of streamer formation in sprites. Geophys. Res. Lett., 34, L06804, doi: 10.1029/2006GL027854. [Link]

Mende, S. B., R. L. Rairden, G. R. Swenson, and W. A. Lyons, 1995: Sprite spectra; N21 PG band identification. Geophys. Res. Lett., 22, 2633-2636, doi: 10.1029/95g102827. [Link]

Minschwaner, K., G. P. Anderson, L. A. Hall, and K. Yoshino, 1992: Polynomial coefficients for calculating $\mathrm{O}_{2}$ Schumann-Runge cross sections at $0.5 \mathrm{~cm}^{-1}$ resolution. J. Geophys. Res., 97, 10103-10108, doi: 10.1029/92JD00661. [Link]

Moudry, D. R., H. C. Stenbaek-Nielsen, D. D. Sentman, and E. M. Wescott, 2002: Velocities of sprite tendrils. Geophys. Res. Lett., 29, doi: 10.1029/2002GL015682. [Link]

Moudry, D. R., H. Stenbaek-Nielsen, D. Sentman, and E. Wescott, 2003: Imaging of elves, halos and sprite initiation at $1 \mathrm{~ms}$ time resolution. J. Atmos. Sol.-Terr. Phys., 65, 509-518, doi: 10.1016/S1364-6826(02)00323-1. [Link]

Naghizadeh-Kashani, Y., Y. Cressault, and A. Gleizes, 2002: Net emission coefficient of air thermal plasmas. J. Phys. D: Appl. Phys., 35, 2925-2934, doi: 10.1088/0022-3727/35/22/306. [Link]

Parra-Rojas, F. C., M. Passas, E. Carrasco, A. Luque, I. Tanarro, M. Simek, and F. J. Gordillo-Vázquez, 2013: Spectroscopic diagnostics of laboratory air plasmas as a benchmark for spectral rotational (gas) temperature determination in TLEs. J. Geophys. Res., 118, 46494661, doi: 10.1002/jgra.50433. [Link]

Pasko, V. P., U. S. Inan, and T. F. Bell, 1998: Spatial structure of sprites. Geophys. Res. Lett., 25, 2123-2126, doi: 10.1029/98g101242. [Link]

Passas, M., J. M. Madiedo, and F. J. Gordillo-Vázquez, 2016: High resolution spectroscopy of an Orionid meteor from 700 to $800 \mathrm{~nm}$. Icarus, 266, 134-141, doi: 10.1016/j.icarus.2015.11.020. [Link]

Sentman, D. D., H. C. Stenbaek-Nielsen, M. G. McHarg, and J. S. Morrill, 2008: Plasma chemistry of sprite streamers. J. Geophys. Res., 113, D11112, doi: 10.1029/2007jd008941. [Link]

Stanley, M., P. Krehbiel, M. Brook, C. Moore, W. Rison, and B. Abrahams, 1999: High speed video of initial sprite development. Geophys. Res. Lett., 26, 32013204, doi: 10.1029/1999GL010673. [Link]

Stenbaek-Nielse, H. and M. G. McHarg, 2004: Sprite Spectra at $1000 \mathrm{fps}$, American Geophysical Union, Fall Meeting 2004, abstract \#AE51A-08.

Stenbaek-Nielsen, H. C., T. Kanmae, M. G. McHarg, and R. Haaland, 2013: High-Speed Observations of Sprite Streamers. Surv. Geophys., 34, 769-795, doi: 10.1007/ 
s10712-013-9224-4. [Link]

Verronen, P. T., E. Kyrölä, J. Tamminen, B. Funke, S. Gil-López, M. Kaufmann, M. López-Puertas, T.v. Clarmann, G. Stiller, U. Grabowski, and M. Höpf- ner, 2005: A comparison of night-time GOMOS and MIPAS ozone profiles in the stratosphere and mesosphere. Adv. Space Res., 36, 958-966, doi: 10.1016/j. asr.2005.04.073. [Link] 